\title{
Controlling Liu Chaotic System with Feedback Method and Its Circuit Realization
}

\author{
Mingjun Wang \\ School of Information Engineering, Dalian University, Dalian, China \\ Email:wmjhome@163.com
}

Received 15 October 2015; accepted 4 March 2016; published 7 March 2016

Copyright (C) 2016 by author and Scientific Research Publishing Inc.

This work is licensed under the Creative Commons Attribution International License (CC BY).

http://creativecommons.org/licenses/by/4.0/

(c) (i) Open Access

\begin{abstract}
In the paper, the Liu system with a feedback controller is discussed. The influence of the feedback coefficient of the controlled system is studied through Lyapunov exponents spectrum and bifurcation diagram. Various attractors are demonstrated not only by numerical simulations but also by circuit experiments. Only one feedback channel is used in our study, which is useful in communication. The circuit experiments show that our study has significance in practical applications.
\end{abstract}

Keywords

Liu System, Chaotic Circuit, Chaotic Control, Circuit Realization

\section{Introduction}

In 1990, Ott, Grebogi and Yorke presented the OGY method to control chaos [1]. After their pioneering work, chaotic control has become a focus in nonlinear problems and a lot of work has been done in the field [2]-[4]. Nowadays, many methods have been proposed to control chaos [5] [6]. Generally speaking, there are two kinds of control ways: feedback control and nonfeedback control. Feedback methods [7]-[11] are used to stabilize the unstable periodic orbit of chaotic systems by feeding back their states. Nonfeedback methods [11]-[14] are adopted to change chaotic behaviors by applying perturbations to some parameters or variables. In the paper, we use feedback method to control the dynamic behavior of Liu system. By adjusting feedback coefficient, Liu system can be stabilized at equilibrium point or limit cycle around its equilibrium. Lyapunov exponents spectrum and bifurcation diagram are adopted to analyze the dynamic behavior of the controlled system. Numerical simulations and circuit experiments show the effectiveness of this method.

\section{The Description of Liu System}

Liu system [15] is described as

How to cite this paper: Wang, M.J. (2016) Controlling Liu Chaotic System with Feedback Method and Its Circuit Realization. International Journal of Modern Nonlinear Theory and Application, 5, 40-47. http://dx.doi.org/10.4236/ijmnta.2016.51004 


$$
\left\{\begin{array}{l}
\dot{x}=a(y-x) \\
\dot{y}=b x-k x z \\
\dot{z}=-c z+h x^{2}
\end{array}\right.
$$

When $a=10, b=40, c=2.5, h=4$ and $k=1$, system (1) exhibits a chaotic behavior. Its attractor is shown in Figure 1. The projections of system (1)'s attractor are shown in Figure 2. System (1) has three equilibriums: $S_{0}(0,0,0), S_{1}(5,5,40), S_{2}(-5,-5,40)$.

Considering the voltage restraint of practical electronic components, let

$$
\left\{\begin{array}{l}
x=10 x^{\prime} \\
y=10 y^{\prime} \\
z=10 z^{\prime}
\end{array}\right.
$$

Then in the new coordinate system, system (1) will be described as

$$
\left\{\begin{array}{l}
\dot{x}^{\prime}=a\left(y^{\prime}-x^{\prime}\right), \\
\dot{y}^{\prime}=b x^{\prime}-10 k x^{\prime} z^{\prime}, \\
\dot{z}^{\prime}=-c z^{\prime}+10 h x^{\prime 2} .
\end{array}\right.
$$

System (3) can be seemed as a reduced Liu system and the equilibriums are $S_{0}^{\prime}(0,0,0), S_{1}^{\prime}(0.5,0.5,4)$, $S_{2}^{\prime}(-0.5,-0.5,4)$. The circuit realization of Equation (3) is shown in Figure 3.

In Figure 3, the voltages of $C_{1}, C_{2}, C_{3}$ are used as variables. The relevant function can be described as

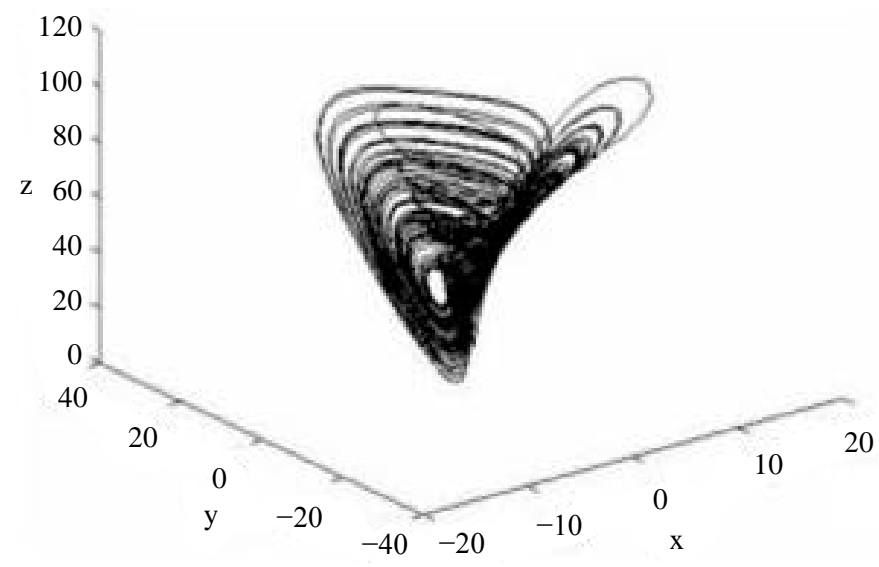

Figure 1. The chaotic attractor of Liu system.

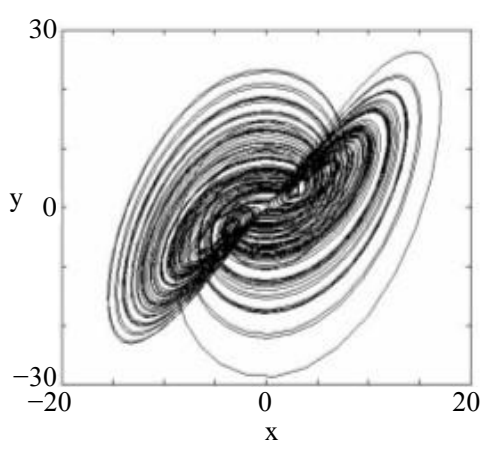

(a)

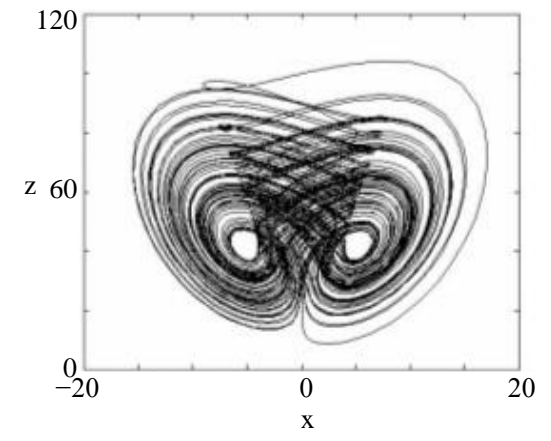

(b)

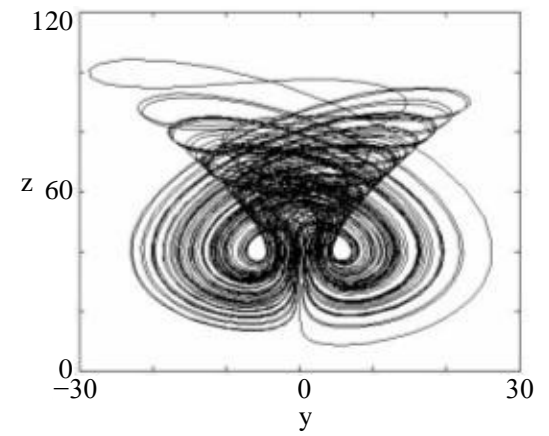

(c)

Figure 2. The projections of Liu attractor. 


$$
\left\{\begin{array}{l}
\dot{x}=\frac{R_{3}}{R_{1} R_{4} C_{1}} y-\frac{R_{6} R_{3}}{R_{5} R_{2} R_{4} C_{1}} x, \\
\dot{y}=\frac{R_{9}}{R_{7} R_{10} C_{2}} x-\frac{R_{9} R_{6}}{R_{5} R_{8} R_{10} C_{2}} x z, \\
\dot{z}=-\frac{R_{12} R_{15}}{R_{13} R_{14} R_{16} C_{3}} z+\frac{R_{12} R_{6}^{2}}{R_{5}^{2} R_{11} R_{13} C_{3}} x^{2} .
\end{array}\right.
$$

When we choose $R_{1}=10 \mathrm{k} \Omega, R_{2}=20 \mathrm{k} \Omega, R_{3}=10 \mathrm{k} \Omega, R_{4}=100 \mathrm{k} \Omega, R_{5}=10 \mathrm{k} \Omega, R_{6}=20 \mathrm{k} \Omega, R_{7}=10 \mathrm{k} \Omega, R_{8}$ $=80 \mathrm{k} \Omega, R_{9}=40 \mathrm{k} \Omega, R_{10}=100 \mathrm{k} \Omega, R_{11}=10 \mathrm{k} \Omega, R_{12}=10 \mathrm{k} \Omega, R_{13}=100 \mathrm{k} \Omega, R_{14}=10 \mathrm{k} \Omega, R_{15}=10 \mathrm{k} \Omega, R_{16}=40$ $\mathrm{k} \Omega, C_{1}=C_{2}=C_{3}=1 \mu \mathrm{F}$, the circuit system (4) is equivalent to system (3). The supplies of all active devices are $\pm 18 \mathrm{~V}$ and the initial voltages of $C_{1}, C_{2}, C_{3}$ are random, we obtain the experiment observations of system (4) as Figure 4 (with Multisim 7.0).

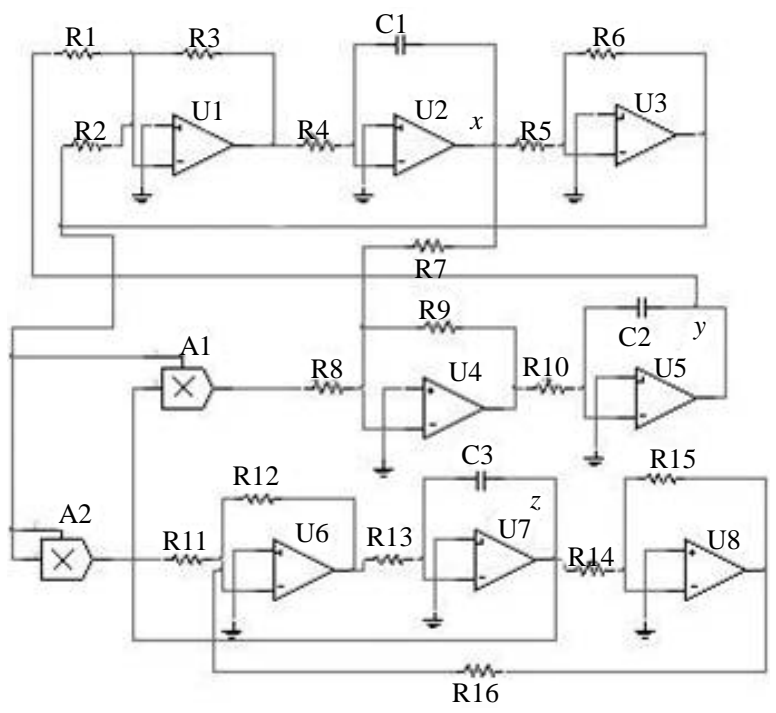

Figure 3. Circuit diagram for system (3).

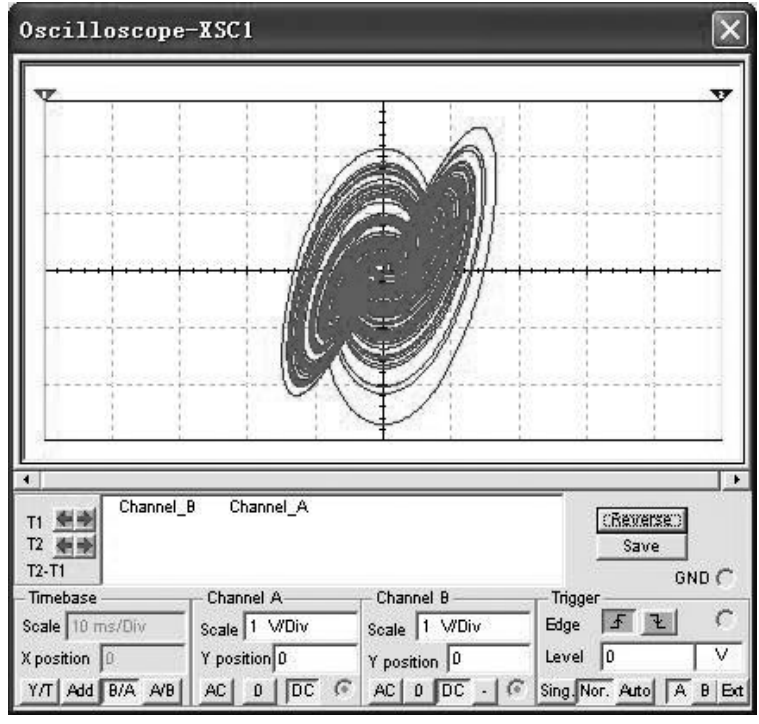

(a)

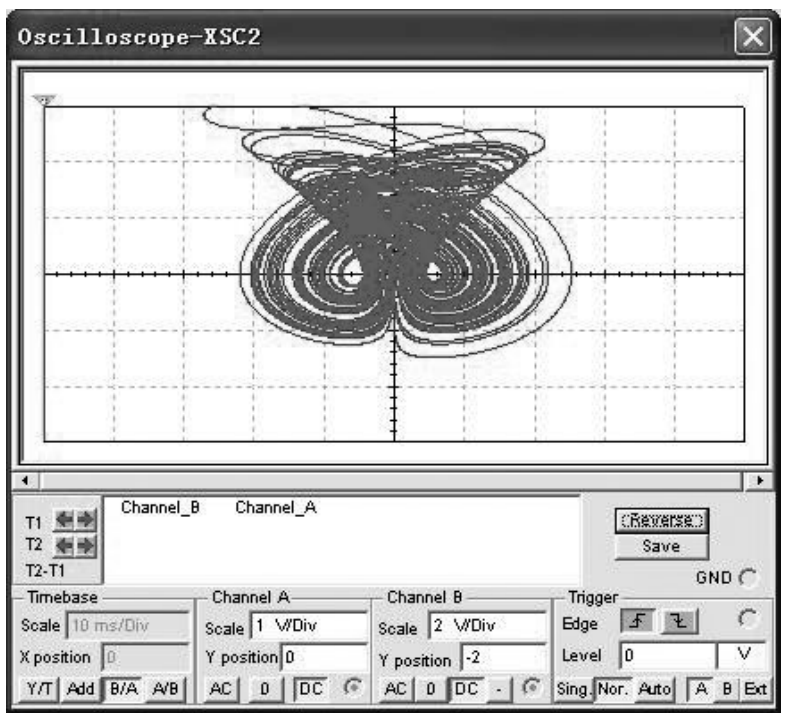

(b)

Figure 4. The experiment observations of system (4). (a) $x$-y plane; (b) $y$-z plane. 
Comparing Figure 2 and Figure 4, we can know that a reduced Liu system has been realized by circuit experiment. Next, we will add a feedback controller to this circuit to control chaos. Various attractors will be demonstrated not only by numerical simulations but also by the circuit experiment observations.

\section{Feedback Control of Liu System}

Suppose we want to stabilize Liu system at equilibrium $S_{1}$ and the limit cycle surrounding $S_{1}$ respectively. For convenience, choose $x$ as feedback variable, this feedback can be added to any of the three functions of Liu system. Applying the controller to the second function, then the controlled Liu system is described as

$$
\left\{\begin{array}{l}
\dot{x}=a(y-x) \\
\dot{y}=b x-k x z-\gamma(x-5), \\
\dot{z}=-c z+h x^{2}
\end{array}\right.
$$

where $\gamma$ is feedback coefficient.

In order to study the relation between $\gamma$ and system (5)'s behavior, we make the bifurcation diagram of system (5) with $0 \leq \gamma \leq 20$ in Figure 5. $X_{\max }$ stands for the largest $x$ in every unsteady period or steady period. When system (5) is stabilized at fixed point or system (5)'s behavior is periodic, $X_{\max }$ has only one value or numbered values with certain $\gamma$; When system (5)'s behavior is chaotic, $X_{\max }$ will have numberless values with certain $\gamma$. According to the method presented by Ramasubramanian et al. [16], we obtain the Lyapunov exponents spectrum of system (5) with $0 \leq \gamma \leq 20$ in Figure 6. When the largest Lyapunov exponent $\lambda_{1}>0$, system (5)'s behavior is chaotic; When $\lambda_{1}=0$, system (5)'s behavior is periodic; When $\lambda_{1}<0$, system (5) is stabilized at fixed point. From Figure 5 and Figure 6, we have the following conclusions: when $\gamma<4.9$, system (5) is chaotic (except a very narrow zone near $\gamma=4.3$, where system (5) may be periodic); when $4.9 \leq \gamma<16.9$, system (5) is periodic; when $\gamma \geq 16.9$, system (5) is stabilized at $S_{1}$.

We obtain the above conclusions by numerical calculation. In fact, the accurate range for $\gamma$ to stabilize system (5) at $S_{1}$ can be obtained by theoretical calculation. Substitute the values of parameters and equilibriums, we obtain the Jacobian matrix of system (5) at $S_{1}(5,5,40)$ :

$$
\boldsymbol{J}\left(S_{1}\right)=\left(\begin{array}{ccc}
-10 & 10 & 0 \\
-\gamma & 0 & -5 \\
40 & 0 & -2.5
\end{array}\right) .
$$

Suppose $\lambda$ as eigenvalue, then the characteristic equation of Equation (6) is

$$
\lambda^{3}+c_{1} \lambda^{2}+c_{2} \lambda+c_{3}=0
$$

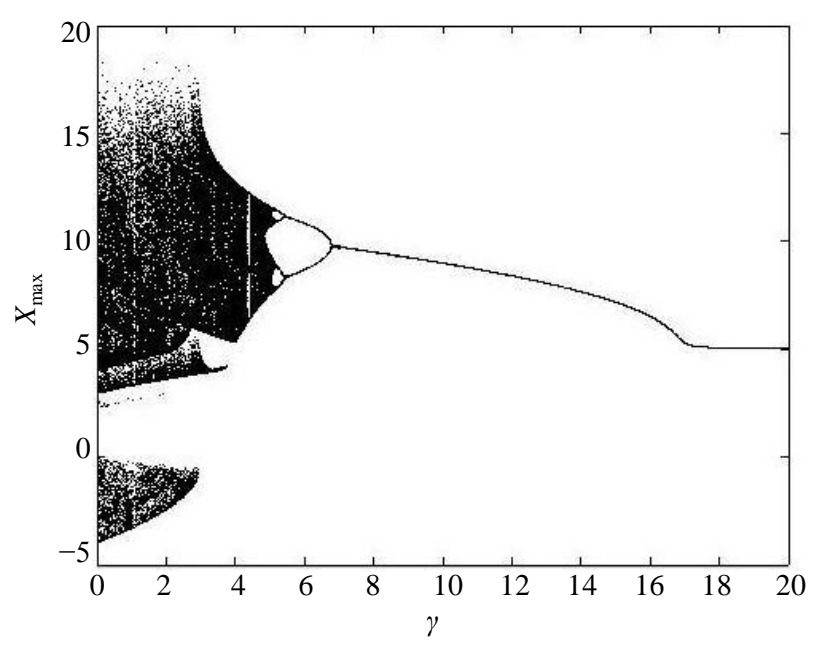

Figure 5. Bifurcation diagram of system (5). 


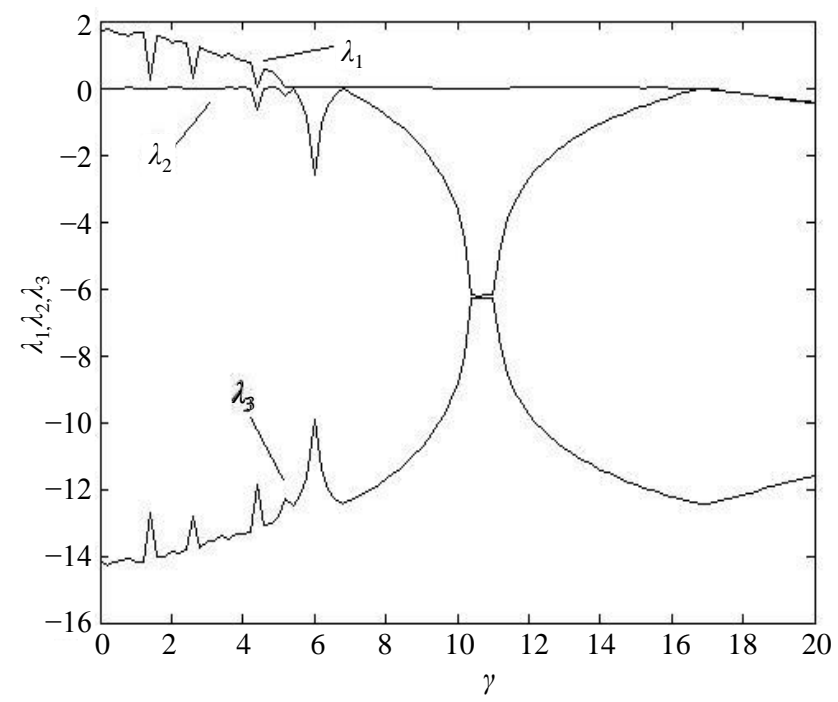

Figure 6. Lyapunov exponents spectrum of system (5).

where $c_{1}=12.5, \quad c_{2}=10 \gamma+25, c_{3}=25 \gamma+2000$.

According to Routh-Hurwitz criterion, when $c_{1}>0, c_{2}>0, c_{3}>0$ and $c_{1} c_{2}-c_{3}>0$, the real parts of all the eigenvalues of Equation (6) are negative, then system (5) will be stabilized at $S_{1}(5,5,40)$. It's easy to obtain the solution $\gamma>16.875$.

\section{Numerical Simulations and Circuit Realization}

As for the reduced Liu system, it's easy to obtain the relevant controlled system:

$$
\left\{\begin{array}{l}
\dot{x}=a(y-x), \\
\dot{y}=b x-10 k x z-\gamma(x-0.5), \\
\dot{z}=-c z+10 h x^{2} .
\end{array}\right.
$$

Obviously the above conclusions about $\gamma$ are still available to system (8). Next we will use system (8) for numerical simulations and circuit experiments. The circuit diagram for system (8) is shown in Figure 7 . The relevant function can be described as

$$
\left\{\begin{array}{l}
\dot{x}=\frac{R_{3}}{R_{1} R_{4} C_{1}} y-\frac{R_{6} R_{3}}{R_{5} R_{2} R_{4} C_{1}} x, \\
\dot{y}=\frac{R_{9}}{R_{7} R_{10} C_{2}} x-\frac{R_{9} R_{6}}{R_{5} R_{8} R_{10} C_{2}} x z-\frac{R_{9}}{R_{19} R_{10} C_{2}}\left(\frac{R_{18}}{R_{17}} x-V_{1}\right), \\
\dot{z}=-\frac{R_{12} R_{15}}{R_{13} R_{14} R_{16} C_{3}} z+\frac{R_{12} R_{6}^{2}}{R_{5}^{2} R_{11} R_{13} C_{3}} x^{2} .
\end{array}\right.
$$

When we choose $R_{17}=R_{18}=10 \mathrm{k} \Omega, V_{1}=0.5 \mathrm{~V}$, all other cognominal electronic components are defined as the above, then circuit system (9) is equivalent to system (8) and we can adjust $R_{19}$ to obtain proper feedback coefficient.

Substitute the value of $R_{17}, R_{18}, R_{9}, R_{10}, C_{2}, V_{1}$, we have

$$
R_{19}=400 / \gamma(\mathrm{k} \Omega) \text {. }
$$

Choose typical value $\gamma=4,6,16,18$ for numerical simulations, the simulation results of system (8) are shown in Figure 8. Choose $R_{19}=100,66.667,25,22.222(\mathrm{k} \Omega)$, we can obtain the equivalent circuit system (9), the experiment results are shown in Figure 9 (with Multisim 7.0). 


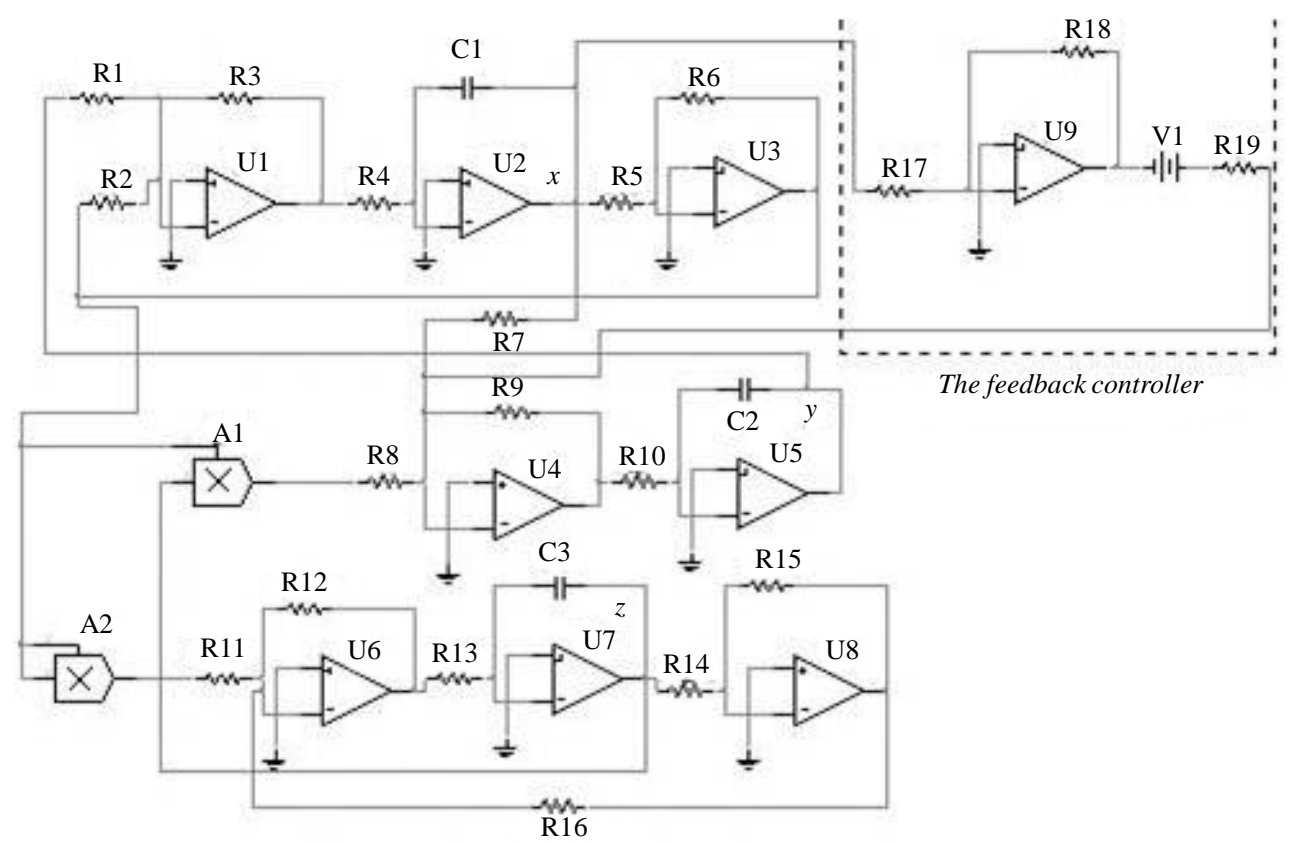

Figure 7. Circuit diagram for system (8).

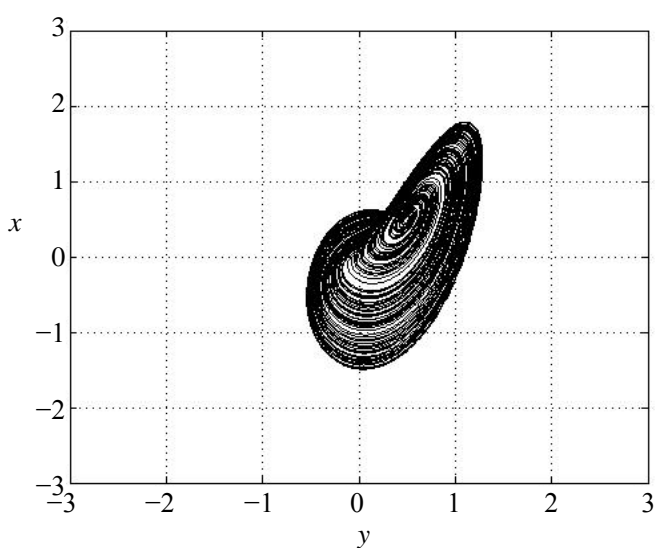

(a)

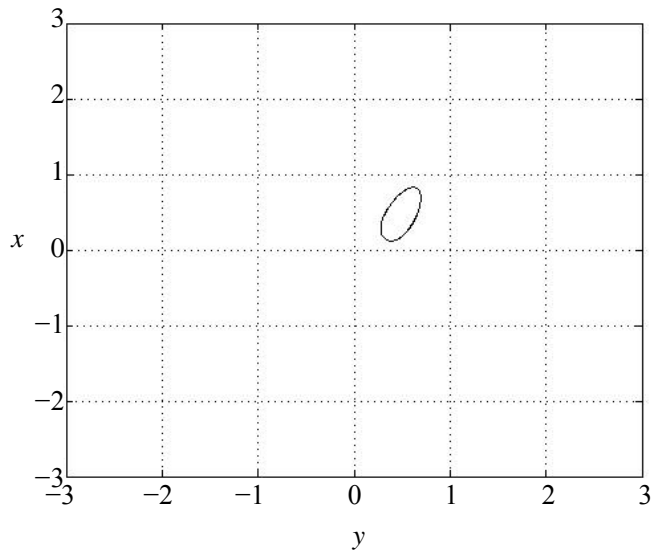

(c)

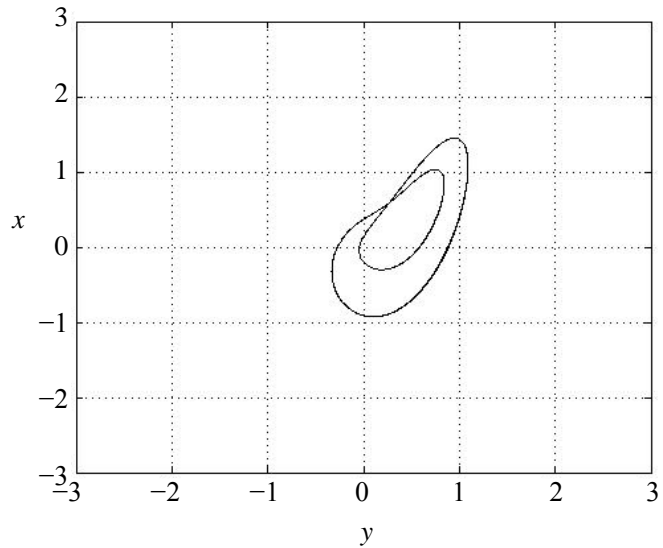

(b)

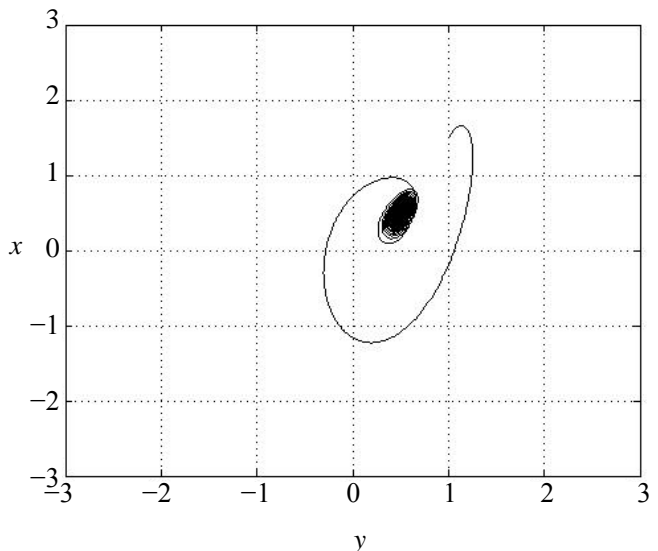

(d)

Figure 8. The simulation results of system (8). (a) $\gamma=4$; (b) $\gamma=6$; (c) $\gamma=16$; (d) $\gamma=18$. 


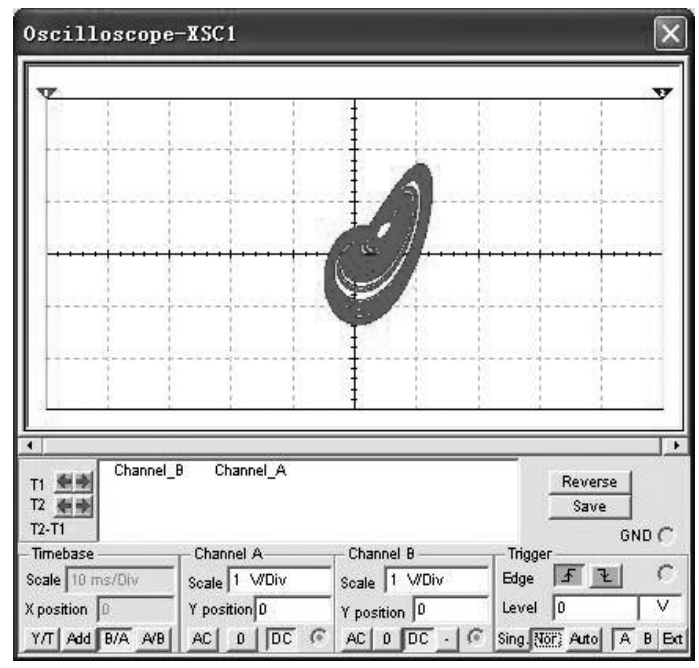

(a)

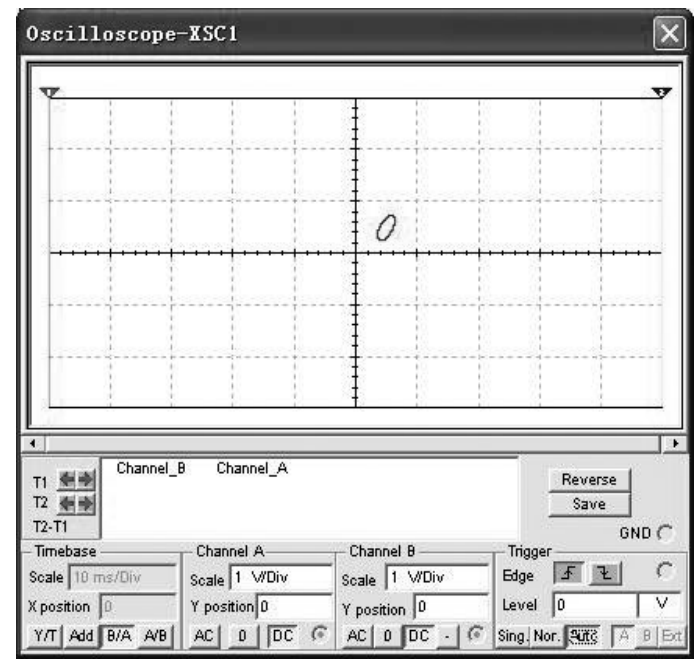

(c)

Figure 9. The experiment observations of system $R_{19}=25(\mathrm{k} \Omega) ;(\mathrm{d}) \quad R_{19}=22.222(\mathrm{k} \Omega)$.

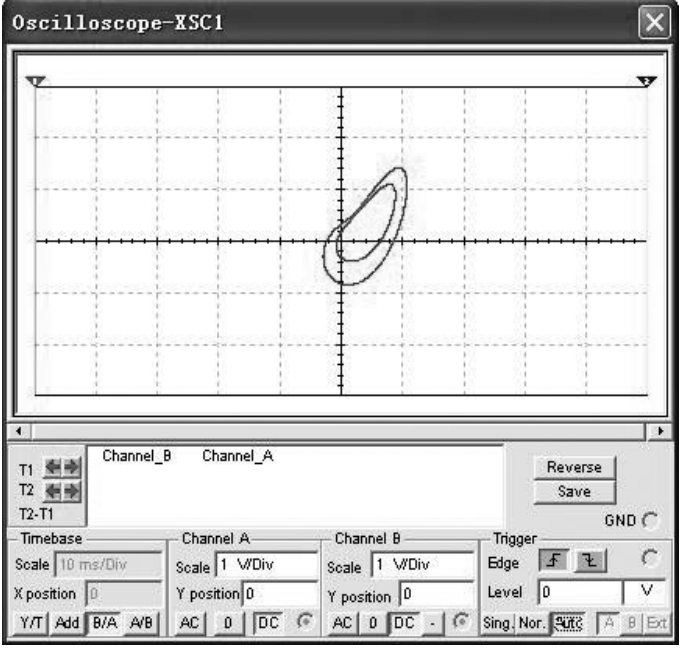

(b)

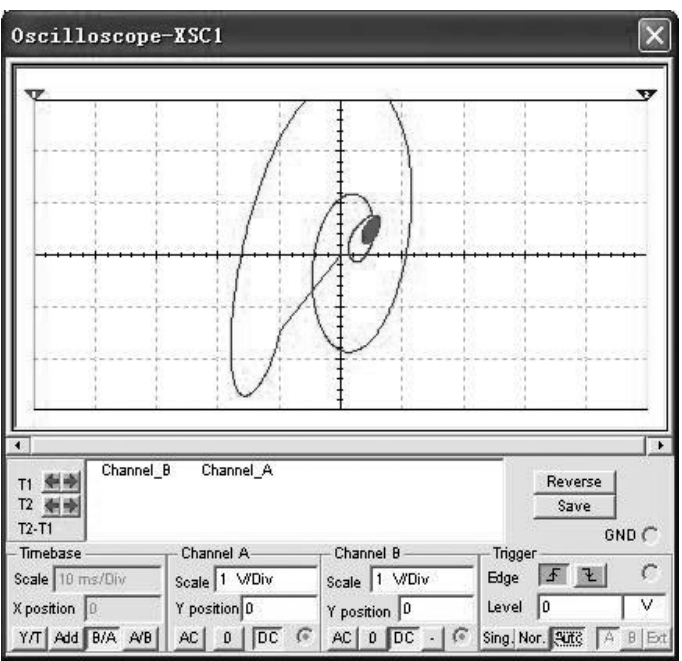

(d)

(9). (a) $R_{19}=100(\mathrm{k} \Omega)$; (b) $R_{19}=66.667(\mathrm{k} \Omega)$; (c)

From Figure 8 and Figure 9, we can know that system (8) is equivalent to system (9) in troth. When $\gamma=4$ ( $R_{19}=100 \mathrm{k} \Omega$ ), the reduced Liu system is chaotic; When $\gamma=6 \quad\left(R_{19}=66.667 \mathrm{k} \Omega\right.$ ), the reduced Liu system is periodic; When $\gamma=16 \quad\left(R_{19}=25 \mathrm{k} \Omega\right)$, the reduced Liu system's behavior is a limit cycle around the equilibrium $(0.5,0.5,4)$; When $\gamma=18\left(R_{19}=22.222 \mathrm{k} \Omega\right)$, the reduced Liu system is stabilized at $(0.5,0.5,4)$ lastly. These results accord with the conclusions in Section 3.

\section{Conclusion}

We study the chaotic control of Liu system with feedback method in the paper. Liu chaotic system and its control are realized not only by numerical simulations but also by circuit experiments. Computer simulation and circuit experiment results show the effectiveness of our method. Moreover, our control needs only one communication channel, which is significant in practical applications.

\section{Acknowledgements}

The work was supported by Doctor Specific Funds of Dalian University. 


\section{References}

[1] Ott, E., Grebogi, C. and Yorke, J.A. (1990) Controlling Chaos. Physical Review Letters, 64, 1196-1199. http://dx.doi.org/10.1103/PhysRevLett.64.1196

[2] Chen, G. and Dong, X. (1998) From Chaos to Order: Methodologies, Perspectives and Applications. World Scientific, Singapore. http://dx.doi.org/10.1142/3033

[3] Wang, G.R., Yu, X.L. and Chen, S.G. (2001) Chaotic Control, Synchronization and Utilizing. National Defence Industry Press, Beijing.

[4] Guan, X.P., Fan, Z.P., Chen, C.L. and Hua, C.C. (2002) Chaotic Control and Its Application on Secure Communication. National Defence Industry Press, Beijing.

[5] Chen, G.R. and Lü, J.H. (2003) Dynamical Analyses, Control and Synchronization of the Lorenz System Family. Science Press, Beijing.

[6] Wang, X.Y. (2003) Chaos in the Complex Nonlinearity System. Electronics Industry Press, Beijing.

[7] Jang, M.J., Chen, C.L. and Chen, C.K. (2002) Sliding Mode Control of Hyperchaos in Rössler Systems. Chaos, Solitons \& Fractals, 14, 1465-1476. http://dx.doi.org/10.1016/S0960-0779(02)00084-X

[8] Ghosh, D., Saha, P. and Chowdhury, A.R. (2010) Linear Observer Based Projective Synchronization in Delay Rössler System. Communications in Nonlinear Science and Numerical Simulation, 15, 1640-1647. http://dx.doi.org/10.1016/j.cnsns.2009.06.019

[9] Zheng, Y.A. (2006) Controlling Chaos Using Takagi-Sugeno Fuzzy Model and Adaptive Adjustment. Chinese Physics, 15, 2549-2552. http://dx.doi.org/10.1088/1009-1963/15/11/015

[10] Gong, L.H. (2005) Study of Chaos Control Based on Adaptive Pulse Perturbation. Acta Physica Sinaca, 54, 35023507.

[11] Roopaei, M., Sahraei, B.R. and Lin, T.C. (2010) Adaptive Sliding Mode Control in a Novel Class of Chaotic Systems. Communications in Nonlinear Science and Numerical Simulation, 15, 4158-4170. http://dx.doi.org/10.1016/j.cnsns.2010.02.017

[12] Paula, A.S. and Savi, M.A. (2011) Comparative Analysis of Chaos Control Methods: A Mechanical System Case Study. International Journal of Non-Linear Mechanics, 46, 1076-1089. http://dx.doi.org/10.1016/j.ijnonlinmec.2011.04.031

[13] Dadras, S. and Momeni, H.R. (2010) Adaptive Sliding Mode Control of Chaotic Dynamical Systems with Application to Synchronization. Mathematics and Computers in Simulation, 80, 2245-2257. http://dx.doi.org/10.1016/j.matcom.2010.04.005

[14] Wu, Z.M., Xie, J.Y., Fang, Y.Y. and Xu, Z.Y. (2007) Controlling Chaos with Periodic Parametric Perturbations in Lorenz System. Chaos, Solitons \& Fractals, 32, 104-112. http://dx.doi.org/10.1016/j.chaos.2005.10.060

[15] Liu, C.X., Liu, T., Liu, L. and Liu, K. (2004) A New Chaotic Attractor. Chaos, Solitons \& Fractals, 22, $1031-1038$. http://dx.doi.org/10.1016/j.chaos.2004.02.060

[16] Ramasubramanian, K. and Sriram, M.S. (2000) A Comparative Study of Computation of Lyapunov Spectra with Different Algorithms. Physica D, 139, 72-86. http://dx.doi.org/10.1016/S0167-2789(99)00234-1 